\title{
Normes environnementales, transferts de gestion et recompositions territoriales en pays betsileo (Madagascar)
}

\section{La gestion contractualisée des forêts}

\author{
Chantal Blanc-Pamard ${ }^{\mathrm{a}}$, Hervé Rakoto Ramiarantsoa ${ }^{\mathrm{b}}$ \\ a Géographe, CEAf, CNRS/EHESS, 96 boulevard Raspail, 75006 Paris, France \\ b Géographe, ICOTEM, MSHS, 99 avenue du Recteur Pineau, 86000 Poitiers cedex, France
}

\begin{abstract}
À partir d'une étude réalisée dans deux terroirs malgaches, les auteurs éclairent les contradictions des discours sur les démarches participatives pour la gestion durable des ressources naturelles ainsi que les ajustements qui en découlent au sein des dispositifs de mise en œuvre. L'ignorance des savoirs locaux et des formes d'organisation produit des territoires conflictuels, ce qui constitue un réel défi pour une gestion de l'environnement prenant en compte les configurations sociospatiales. La confrontation entre les dynamiques locales et les normes internationales entraîne une requalification des savoirs, des pouvoirs et des territoires. Plus largement, cette analyse questionne la conception de politiques environnementales et leur capacité à «gouverner l'environnement».
\end{abstract}

La Rédaction

\section{Mots-clés :}

Madagascar ; corridor

forestier; territoire ; politique environnementale ; gestion contractuelle

\section{Keywords:}

Madagascar;

forest

corridor; territory;

environmental policy;

contractual

management
Résumé - Depuis les années 1990, Madagascar a adopté une politique de conservation de la biodiversité qui se traduit par la mise en place de dispositifs territoriaux. L'innovation institutionnelle que représente la gestion locale des ressources renouvelables coexiste désormais avec le modèle ancien et expansionniste des aires protégées. Cette politique environnementale entraîne des réglementations portant sur des espaces physiques, mais aussi sur les droits d'usage, d'accès et de commercialisation, d'où des enjeux d'appropriation de l'espace et des ressources et des conflits autour des droits d'accès et d'usage. Notre étude en pays betsileo porte sur deux territoires ruraux inscrits dans des processus de reconfiguration liés à des transferts de gestion des ressources naturelles renouvelables (TGRNR) sur le versant ouest du corridor forestier. Les réglementations environnementales corsètent les paysans et leurs activités d'un faisceau de contraintes et créent une requalification des territoires, des savoirs et des pouvoirs. L'analyse des réponses des acteurs questionne la conception territoriale d'une politique de préservation de la biodiversité.

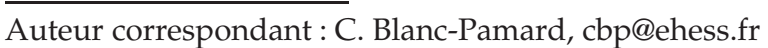


La mégabiodiversité malgache se caractérise par un ensemble d'écosystèmes remarquables, au degré d'endémisme de $80 \%$ pour la faune et de $85 \%$ pour la flore. Conservation International classe Madagascar, avec les îles de l'océan Indien, parmi les 35 biodiversity hotspots prioritaires pour la conservation des ressources naturelles ${ }^{1}$. De ce fait, la dégradation environnementale apparaît comme une forte menace pour l'île, mais aussi pour la planète. Madagascar est considéré par la communauté internationale comme l'un des pays où le patrimoine biologique exceptionnel est le plus en danger $^{2}$. Les préoccupations environnementales ne cessent de prendre de l'importance dans la conception de la politique publique pour préserver et valoriser cette mégabiodiversité (Marcus et Kull, 1999 ; Blanc-Pamard et Rakoto Ramiarantsoa, 2003; Andriananja et Raharinirina, 2004). Différentes conventions internationales relatives à l'environnement ont été ratifiées et la déclaration de Durban ${ }^{3}$, en 2003, indique la prise de position pour renforcer les perspectives de conservation.

Sur la Grande Île, la conscience publique et officielle des perturbations environnementales s'est manifestée dès le XIXe siècle $^{4}$. Différentes mesures de défense de la forêt ont été mises en place dès la colonisation, conçues par les seuls services techniques qui considéraient les modes de gestion traditionnels comme «destructeurs de l'environnement ».

Une modification intervient dans les années 1990, dans un contexte de libéralisation et de démocratisation. Les stratégies centralisées, jugées inefficaces, sont remplacées par des approches participatives. De nouvelles règles institutionnelles accompagnent une décentralisation ainsi que l'implication des populations dans la gestion des ressources. Les changements entraînés par l'inclusion de la dimension de protection de l'environnement au niveau local, avec la mise en œuvre de transferts de la responsabilité de la gestion aux populations, sont au centre de notre propos, plus particulièrement les recompositions introduites par l'interférence de ces territoires de conservation avec les formes d'organisation sociale et territoriale en place. Notre réflexion montre, à partir de l'étude de deux territoires ruraux inscrits dans des processus de reconfiguration territoriale liés à une politique

\footnotetext{
${ }^{1}$ Ce texte a fait l'objet d'une présentation au séminaire de l'ATI (action thématique interdépartementale) « Aires protégées » de l'IRD en novembre 2005, à Ouagadougou.

2 Voir à ce sujet la synthèse établie par Goodman et Benstead (2003).

${ }^{3}$ Lors du $5^{\text {e }}$ Congrès sur les Parcs, à Durban (Afrique du Sud, septembre 2003), le président de la République Marc Ravalomanana s'est engagé à tripler la surface des aires protégées, de 1,7 à 6 millions d'hectares en 5 ans.

4 À l'époque des royaumes merina, la détermination de protéger les forêts est réelle. Elle se traduit par les édits d'Andrianampoinimerina (1787-1810) et le code des « 305 articles » (1881).
}

environnementale de transfert de gestion ${ }^{5}$, comment les dispositifs négligent les représentations, les valeurs et les logiques des différents acteurs en interaction, en particulier les pratiques locales et le foncier. Il s'agit de souligner les conséquences des enjeux de conservation de la biodiversité et des dispositifs mis en place à cet effet sur la requalification des savoirs, des pouvoirs et des territoires.

\section{D'une protection de la nature à une protection de l'environnement}

La déforestation est une question récurrente et une préoccupation ancienne (Jarosz, 1996). Les réponses apportées montrent une évolution dans les dispositifs, de la protection de la nature à la conservation de la biodiversité (Ramamonjisoa, 2004). Depuis le XIXe siècle, des réglementations sont élaborées en se référant à un lieu, à un type d'action (reboisement), à une entité naturelle (forêt). Aujourd'hui, elles portent sur les «corridors", et plus précisément sur le « corridor forestier de Fianarantsoa », site de notre étude.

Sous la colonisation française, le premier département technique créé par Gallieni est le Service forestier (Fremigacci, 1998), dont l'objectif, en reboisant, est de réduire l'érosion due à la dénudation avancée du relief. Les feux de végétation, un autre aspect de la perturbation écologique, font l'objet d'une très forte répression, ce qui traduit une conception ignorant les sociétés, les populations étant accusées de détruire leur propre environnement ${ }^{6}$. Parallèlement au reboisement et à la lutte contre les feux, les dix premières réserves naturelle $(\mathrm{RN})$ sont mises en place en 1927. Elles sont choisies dans différentes situations écologiques. Cette initiative fait de Madagascar l'un des premiers pays établissant un système de réserves naturelles à l'échelle nationale.

Le dispositif protectionniste est complété dans les premières années de l'indépendance par d'autres catégories d'aires protégées $(\mathrm{AP})$ : parc national, réserve spéciale, forêt classée, réserve de chasse, périmètre de reboisement et de restauration. Hors de ces zones à statut spécial, et sur les terrains domaniaux, la direction des Eaux et Forêts veille à faire respecter les mesures de protection. Cette phase de politique de protection a connu un flottement,

\footnotetext{
${ }^{5}$ Chercheurs associés à l'UR 168 (Dynamiques environnementales entre forêt, agriculture et biodiversité) de l'IRD, nous menons nos recherches dans le cadre du programme GEREM (Gestion des espaces ruraux et environnement à Madagascar). Ce programme a été mis en place, en juin 2003, en pays betsileo, dans la commune rurale d'Androy, sur le versant ouest du corridor forestier. Il est conduit en partenariat entre l'IRD et le CNRE (Centre national de recherches sur l'environnement).

6 Déjà le code des « 305 articles » indique que celui qui est pris en flagrant délit de feu en forêt est rabaissé au rang de serviteur.
} 
dans les années 1970, dans un contexte national caractérisé par une crise politique, économique et financière.

Les années 1980 sont caractérisées par une politique conservationniste qui impose 1'approche PCDI (projet de conservation et développement intégré), alors que de nouvelles aires protégées sont créées. Les PCDI proposent le zonage d'un espace délimité, divisé entre une aire centrale de protection intégrale et une zone périphérique aux différents degrés d'accessibilité pour les populations. Cette approche - qui s'accorde avec la conception des experts de la Banque mondiale - considère que la pauvreté est «à l'origine des pressions exercés sur l'environnement » et qu'elle est un facteur essentiel de la dégradation (Cleaver et Shreiber, 1994). Les résultats des PCDI sont de portée limitée. Ils ont mal fonctionné et ont eu des effets contraires en considérant d'emblée les populations locales comme de «dangereux destructeurs de l'environnement ».

La décennie 1990 marque une évolution des politiques de gestion des ressources naturelles par des actions qui associent information et formation, avec un objectif de gestion durable des forêts. En 1990, Madagascar est le premier pays du continent africain à avoir adopté une charte sur l'environnement. La même année, le pays s'accorde avec les grands bailleurs de fonds (Banque mondiale, United States Agency for International Development [USAID], Coopération française) pour se doter d'un plan d'action environnemental (PAE) sur 15 ans. Par ailleurs, la décentralisation, relancée, intervient par un transfert des responsabilités de gestion des ressources naturelles aux communautés locales, ce qui engendre de nouvelles réglementations pour les droits d'accès et d'usage des ressources et du foncier.

Le PAE qui a démarré en 1990 a dû évoluer après un bilan de la première phase. Le premier plan environnemental (PE I), en effet, présentait des aspects exagérément conservationnistes. Ainsi, Madagascar s'est doté dans la deuxième phase du PAE d'un cadre juridique de cette décentralisation (Aubert, 2002). La loi dite GELOSE (gestion locale sécurisée) de 1996 entre dans ce processus. Elle concerne un ensemble de ressources renouvelables domaniales (forêts, eau, terres de parcours, etc.) dont une politique nationale accorde la gestion aux communautés locales concernées. La sécurisation foncière relative (SFR) qui accompagne la GELOSE constitue une autre innovation institutionnelle. Une formule spécifiquement forestière et plus souple, la gestion contractualisée des forêts (GCF), a été mise en place par décret en 2001 (ibid.). GELOSE et GCF sont les deux formes de transfert de gestion des ressources naturelles renouvelables (TGRNR) proposées aux communautés paysannes locales.

L'innovation institutionnelle que représente la gestion locale des ressources renouvelables coexiste désormais avec le modèle ancien et expansionniste des aires protégées, ce qui entraîne une concurrence pour le contrôle du territoire. Ces diverses politiques de conservation de la biodiversité ont des logiques spécifiques et produisent des organisations territoriales et sociales différentes; elles bouleversent les modes locaux de gestion de l'accès aux ressources et de leur usage.

La politique de gestion environnementale à support territorial se traduit non seulement par la coexistence de différentes formes de gestion, mais aussi par une imbrication des espaces à protéger, à différentes échelles : du hotspot à la bande de vingt mètres au-dessus des basfonds rizicoles, en passant par les écorégions, les parcs nationaux, les forêts contractualisées (GCF et GELOSE), les territoires de développement et de conservation (TDC), les sites de conservation (SDC). La délimitation d'espaces à protéger de l'érosion de la biodiversité constitue à tous les niveaux une trajectoire d'enclosures ${ }^{7}$. L'enclosure des forêts entraîne soit leur confiscation, soit une réglementation des droits d'usage, d'accès, de production, de commercialisation.

Les aires protégées recouvrent en $20063 \%$ du territoire malgache, ce qui correspond à un taux faible si l'on se réfère au ratio de $10 \%$ que l'UICN considère comme raisonnable pour chaque pays du monde (Blanc-Pamard et Rakoto Ramiarantsoa, 2003). En ce qui concerne les contrats de TGRNR (GELOSE et GCF), présentés comme « des piliers du développement durable à Madagascar», 500 sont en vigueur en 2005 dans les six provinces de la Grande Île et une centaine en cours de traitement (PE III, 2005). Ces contrats couvrent une superficie de 500000 ha, soit une moyenne de 1000 ha par transfert, et touchent 50000 ménages, soit 2,5\% de la population rurale du pays; $70 \%$ portent sur des forêts, $20 \%$ sur des ressources aquatiques, $3 \%$ sur des pâturages et $7 \%$ sur des ressources diversifiées (ibid.). Le contrat GCF, moins contraignant que le contrat GELOSE, connaît un succès relatif (Rakoto Ramiarantsoa et Samyn, 2004).

Cette évolution d'ensemble s'inscrit dans le mouvement d'idées qui relie les questions d'environnement et de développement, et qui a abouti aux résolutions du premier Sommet de la Terre tenu à Rio en 1992 (Ramamonjisoa, 2004). La politique de transfert de gestion aux communautés rurales marque par ailleurs un changement délicat en tentant d'instaurer les conditions institutionnelles d'une gestion partagée des ressources naturelles, alors qu'une telle disposition ne fait pas partie de la culture des sociétés rurales malgaches (BlancPamard et Fauroux, 2004). Enfin, il faut souligner que la politique environnementale malgache n'a pas seulement à voir avec la protection des biotopes, mais aussi avec des constructions politiques entre Hautes Terres et Basses Terres (zones côtières), les zones à conserver étant de faible superficie sur les Hautes Terres et le groupe Vision

\footnotetext{
7 Jean-Pierre Berlan (2002) qualifie d' « enclosures du vivant » la protection des plantes cultivées.
} 
Durban $^{8}$, un moyen de rééquilibrage géopolitique ${ }^{9}$. Le corridor forestier relève de ce contexte.

\section{La gestion contractualisée des forêts (GCF) : deux cas d'étude}

Notre étude concerne deux cas de gestion contractualisée des forêts situés dans le nord du massif forestier appelé « corridor » et sur son versant ouest ${ }^{10}$. Ils sont dans la zone périphérique du Parc national de Ranomafana, créé en 1991. Récents, car le transfert de gestion date de 2003, ils représentent un exemple de gouvernance environnementale locale visant à permettre aux communautés riveraines de contribuer, de façon légitime et représentative, à la réduction des principaux facteurs de dégradation. Ces dispositifs territoriaux de conservation de la biodiversité interfèrent avec les formes d'organisation territoriale et sociale en place, ce qui pose la question de leur appropriation dans un contexte local.

\section{Le « corridor forestier de Fianarantsoa »}

La décennie 1990 a vu le développement de l'approche « corridor forestier» (corridor biologique ou écologique) lors de réunions internationales sur les questions d'environnement, en raison du constat du morcellement ou de la fragmentation qui menace les écosystèmes forestiers ${ }^{11}$. Un nouveau concept de gestion, le " corridor ", a été créé en vue d'une meilleure conservation de la biodiversité et a été mis en place à différentes échelles dans les pays du Nord et les pays du Sud. Ainsi, le vestige du massif forestier en bordure orientale des Hautes Terres est désormais appelé " corridor».

Le concept de corridor, dans le contexte de Madagascar, a été proposé en avril 1995 pendant l'Atelier scientifique sur la définition des priorités de conservation de la diversité biologique à Madagascar ${ }^{12}$

\footnotetext{
${ }^{8}$ Groupe de travail mis en place suite à la déclaration du président de la République à Durban, en septembre 2003 ( $c f$. supra, note 3).

9 Voir à ce sujet l'article de Giraut et al. (2004), qui souligne que les dispositifs spatiaux des parcs et réserves renvoient en Afrique à des fonctions géopolitiques.

10 Sur le versant nord-ouest, en pays betsileo, on compte un autre transfert de gestion à Ranomena.

11 Corridor : «Unité paysagique qui tranche dans l'espace environnant en raison de sa configuration linéaire relativement étroite, de caractère végétal (haie, ripisylve, trouée...) ou topographique (vallon, cours d'eau...) et qui relie d'autres unités plus massives mais de nature analogue, en créant une continuité qui permet aux espèces animales de circuler ou aux végétaux de se propager de l'une à l'autre sans devoir s'exposer à un milieu plus hostile» (Da Lage et Métailié, 2000). Voir aussi Forman et Godron (1981), ainsi que Burel et Baudry (1999).

12 Madagascar a procédé à la ratification de la Convention sur la diversité biologique en 1995 et en est devenue partie contractante en 1996.
}

(Rakotosamimanana et Ganzhorn, 1995). Lors de cet atelier qui a défini une approche écorégionale, le corridor forestier ou corridor RAI (Ranomafana-AndringitraIvohibe) entre le Parc national de Ranomafana au nord et la Réserve spéciale du pic d'Ivohibe en passant par le Parc national d'Andringitra (Fig. 1), fut identifié comme ayant un intérêt biologique exceptionnel et reconnu comme prioritaire en termes de préservation (CarrièreBuchenschutz, 2006).

L'USAID $^{13}$ (projet «Landscape Development Interventions », LDI) et Conservation International (CI) ont, dès le démarrage, fait porter leurs actions sur ce corridor avec l'objectif de préserver « un massif forestier riche en biodiversité et menacé par les pressions humaines ». Dans les documents du LDI, la présentation du contexte est la suivante :

"Ce qui est appelé le "corridor" est le dernier vestige d'une vaste forêt naturelle qui se trouvait autrefois le long de l'escarpement longitudinal que sépare la côte est des Hautes Terres. Actuellement, le corridor n'est qu'une bande étroite (de 5 à $15 \mathrm{~km}$ de largeur) de forêt qui se trouve au sud du Parc national de Ranomafana et au nord du Parc national d'Andringitra. Cette forêt joue un rôle primordial non seulement pour les écologues, mais aussi pour les populations locales » (Freudenberger et Freudenberger, 2002).

La forêt est décrite plus par sa géométrie (un rectangle allongé $\mathrm{N}-\mathrm{S})$, sa superficie $\left(9000 \mathrm{~km}^{2}\right.$ environ), sa largeur (en diminution), que par sa structure interne. Sa dégradation de part et d'autre, à l'ouest comme à l'est, lui confère en fait une réalité. Cette logique de corridor repose sur une entité considérée comme homogène d'un point de vue biogéographique.

\section{Des transferts de gestion des ressources naturelles renouvelables (TGRNR)}

Sur le versant ouest du corridor forestier betsileo : deux GCF mitoyennes

Depuis le début des années 1990, une requalification des territoires de deux types (Parc national de Ranomafana, réserve exclusive et espace confisqué, créé en 1991 sur le modèle ancien des aires protégées, et TGRNR, innovation institutionnelle mettant en place un mode de gestion local depuis 2001) affecte le versant ouest du corridor betsileo, dans sa partie nord.

\footnotetext{
13 L'USAID finance le programme LDI, qui a fonctionné de juin 1998 à décembre 2003. LDI est devenu PTE (Programme de transition écorégionale) de janvier 2004 à septembre 2004, puis ERI (Eco-Regional Initiatives) à partir de septembre 2004 pour une durée de 5 ans. Le programme LDI est l'un des grands programmes financés pour soutenir le PE II. Le programme ERI est un soutien au PE III.
} 


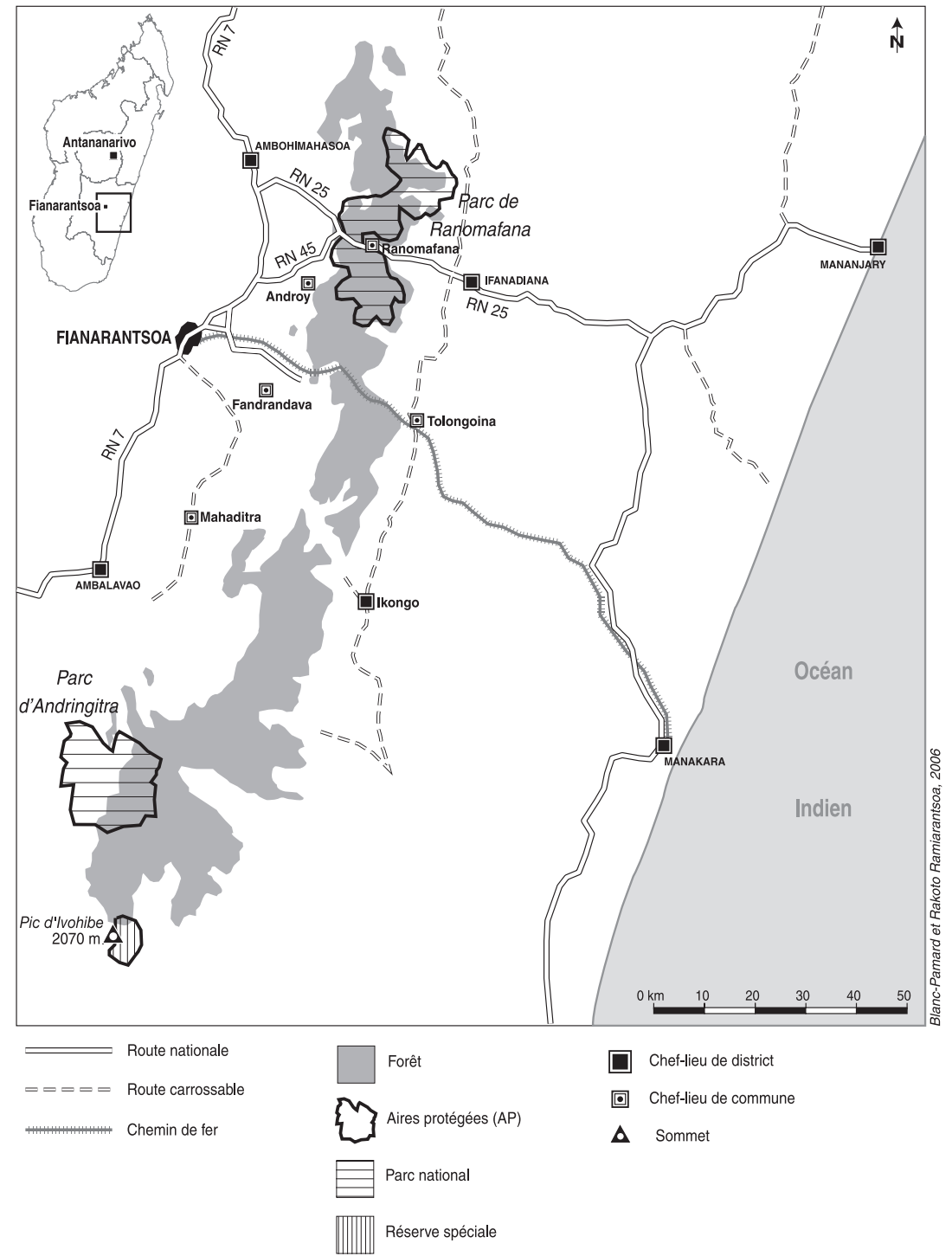

Entre les trois aires protégées (AP), le Parc national de Ranomafana (41 601hectares) créé en 1991, à 120 km environ du Parc

national d'Andringitra ( $31160 \mathrm{ha}$ ) inauguré en 1999 , et la Réserve spéciale du pic d'lvohibe datant de 1964 , à une vingtaine
de kilomètres des limites sud du Parc d'Andringitra, un couloir forestier d'une longueur approximative de $180 \mathrm{~km}$ permet une liaison.

Fig. 1. Le corridor forestier Ranomafana-Andringitra-Ivohibe (RAI).

Notre étude sur ce versant ouest concerne deux villages de la commune rurale d'Androy : Ambendrana, en situation de lisière, et Amindrabe, localisé en forêt (Fig. 2). Ces deux villages sont dans la zone périphérique du Parc national de Ranomafana et sont chacun concernés par un transfert de gestion au bénéfice des acteurs regroupés dans une communauté locale de base (COBA : groupements volontaires d'individus réunis dans une association et unis par les mêmes intérêts). L'inventaire, la délimitation et le zonage des espaces à protéger définissent deux territoires de GCF mitoyens en forêt, l'un de 5212 ha, l'autre de 1496 ha. Ambendrana, un terroir alliant espace ouvert et espace sous forêt, et Amindrabe, un territoire entièrement en forêt, se rejoignent sur un point : l'enriziculture prioritaire. L'installation de la riziculture anticipe l'occupation des versants selon des pratiques qui allient logique écologique, stratégies foncières et régulation sociale.

Le dispositif GCF est régi par un décret de 2001. La gestion communautaire se fait dans le cadre d'une COBA. L'exploitation de la forêt doit s'effectuer conformément à un plan d'aménagement qui fixe le volume annuel de prélèvement et le zonage des unités (zone de conservation et zone de droits d'usage). La gestion contractualisée cible une réduction de la pression sur le corridor avec quelques thèmes d'intervention (cultures en courbes de niveau, plantation de vétiver, pépinières de caféiers et d'orangers, pisciculture, ruches au village...) visant à une intensification agricole.

À Ambendrana, sur une partie du fokontany (unité administrative de base), la forêt a été découpée en trois zones gérées par une COBA (Fig. 3). Les différentes 


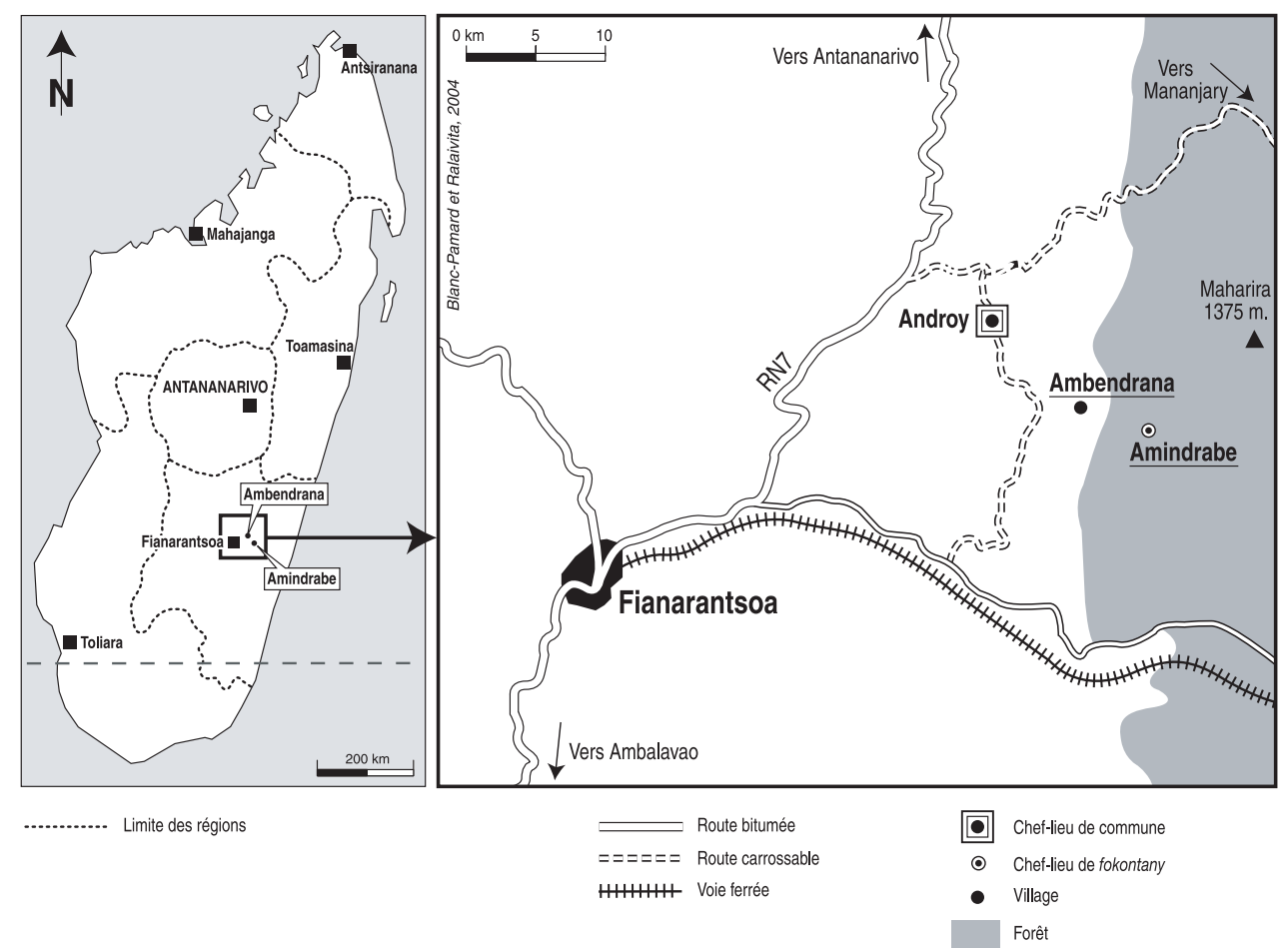

Fig. 2. Carte de localisation.

étapes du processus de contractualisation ont duré plusieurs mois, de la campagne d'information villageoise à la conclusion du contrat. La demande de transfert de gestion a été établie auprès du service des Eaux et Forêts en mai 2002 avec l'appui du LDI. Le contrat de gestion entre l'administration forestière et la communauté de base, ou COBA, a été signé le 28 janvier 2003 (Blanc-Pamard et Ralaivita, 2004).

Pourtant, ce n'est qu'après la signature du contrat que les membres de la COBA disent avoir réalisé que des interdictions accompagnaient la gestion de leur forêt (ala gasy, litt. forêt malgache, forêt naturelle), dont les deux tiers se retrouvent sous surveillance. Par ailleurs, si des impératifs écologiques justifient le zonage lors de l'élaboration du plan d'aménagement, la délimitation ne prend pas réellement en compte la qualité des ressources forestières. En effet, la proportion de forêts sanginafo (soumises à des incendies volontaires) dans la superficie de la forêt contractualisée, notamment dans la partie en zone de protection maximale, s'accorde mal avec l'objectif de conservation de la biodiversité (Fig. 4). De plus, la présence, dans ce périmètre, d'anciens villages (tanàna haolo) dont la création remonte à plus de deux siècles manifeste une utilisation de la forêt qui contredit la représentation que les politiques environnementales ont de celle-ci, c'est-à-dire une forêt riche en biodiversité et dont tout l'intérêt tient à son caractère de forêt primaire.

À Amindrabe, les trois quarts du fokontany sont découpés et gérés de façon contractuelle (GCF) (Fig. 5). Cinq blocs de cantonnement des droits d'usage $\mathrm{e}^{14}$ sont répartis sur le territoire, en raison de la dispersion de l'habitat; ils permettent aux principaux villages de disposer des ressources forestières dans un rayon convenable. La gestion est ainsi délocalisée (Blanc-Pamard et al., 2005). Cette distribution est d'autant plus appropriée que la proximité du Parc national de Ranomafana au nord-est et à l'est interdit l'accès à la forêt et à ses usages locaux. Sur la carte du zonage établie par des membres de la COBA et des techniciens de Conservation International (l'organisme d'appui), dans les cinq zones de droits d'usage, il n'est fait aucune mention de la superficie et de la localisation de la zone de droits d'usage actuels (2003-2005), qui sont pourtant mentionnées dans le contrat. C'est un oubli qui rend peu crédible aux yeux des paysans la gestion de leur forêt et qui introduit une différence entre ce qui a été annoncé et la réalité : «Ce n'est pas un plan d'aménagement de la forêt, mais seulement une délimitation. »

La figure 6 montre une juxtaposition de territoires de conservation, en partie intriqués avec des territoires administratifs. Cette territorialisation dessine un espace de contraintes pour les paysans, qui déploient des stratégies pour adapter leurs propres organisations sociales et les pratiques qui y sont liées à ces nouvelles mailles.

\footnotetext{
14 Accords de droits d'usage aux riverains de ces blocs concernant l'exercice de leurs activités traditionnelles d'exploitation de la forêt selon la réglementation et sans but commercial.
} 


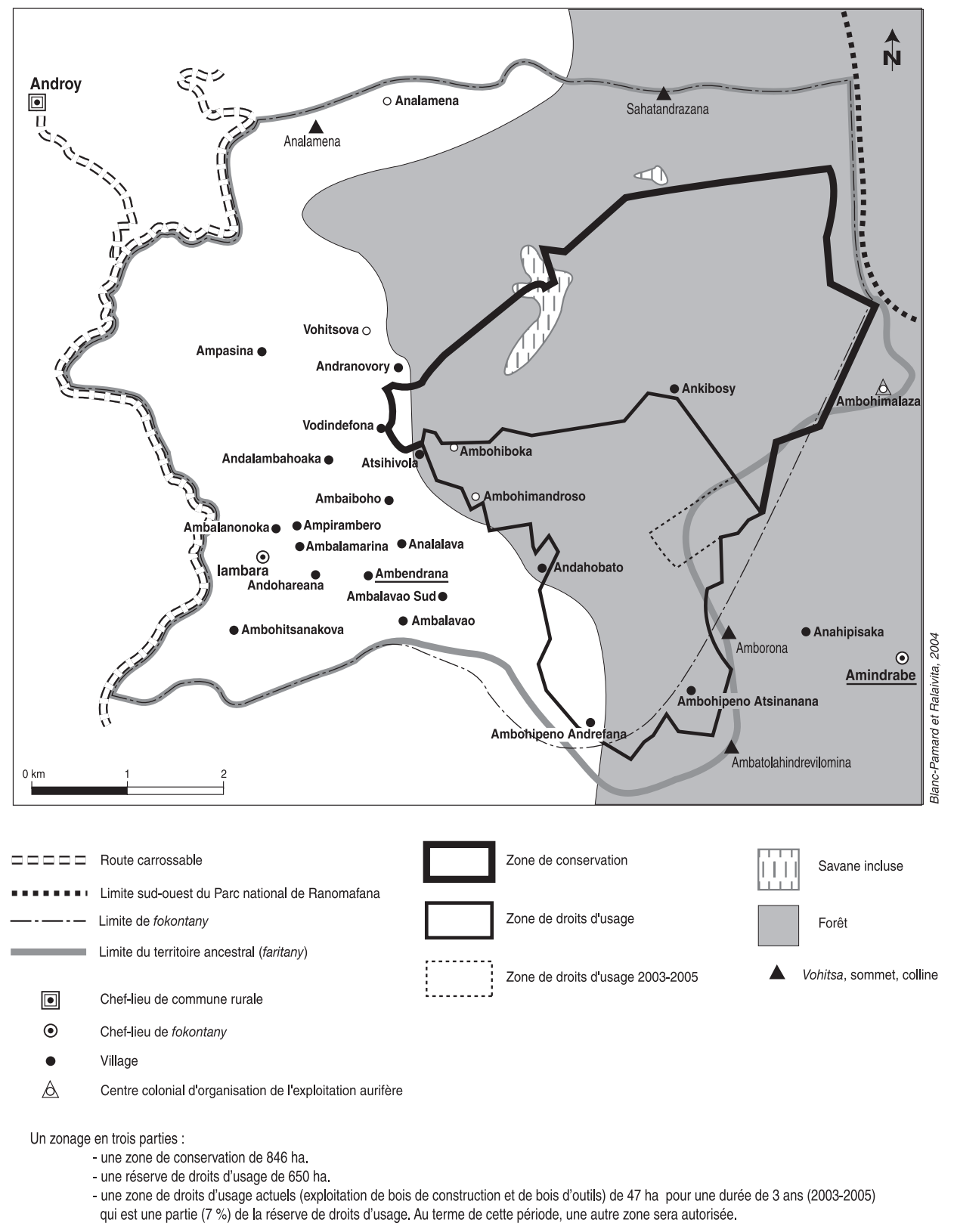

Fig. 3. Le zonage de la forêt gérée par la COBA d'Ambendrana.

\section{Une requalification des territoires, des savoirs et des pouvoirs}

Une distorsion entre le foncier local et le foncier formel caractérise les deux territoires sous GCF. Il ne saurait en être autrement dans la mesure où le programme GCF correspond à une volonté politique de mettre en œuvre un dispositif institutionnel et technique qui se superpose aux trames existantes. La figure 7 traduit un tel décalage sur les territoires d'Ambendrana et d'Amindrabe. Elle montre une organisation du foncier local par groupe de descendants et par bassin versant. En revanche, le foncier formel se superpose et crée des distorsions entre territoires fondés sur des bases écologiques ou sociales, entre accès des différents groupes aux ressources forestières, et même entre les deux GCF. L'interférence des territoires de conservation avec les formes d'organisation sociale et territoriale en place crée une situation conflictuelle.

La situation foncière dans les fokontany d'Ambendrana et d'Amindrabe révèle en outre des enjeux radicalement différents. À Ambendrana, s'observe un contrôle des terres qui se renforce au profit des groupes (ankohonana) fondateurs du village, et plus précisément au bénéfice des deux plus puissants. Leur pouvoir se situe dans une logique de filiation et de résidence. On pourrait les qualifier de «frères voisins » tant la solidité de la gestion des terres repose sur la capacité de contrôle horizontal, de quadrillage social. Les leaders investissent toutes les 


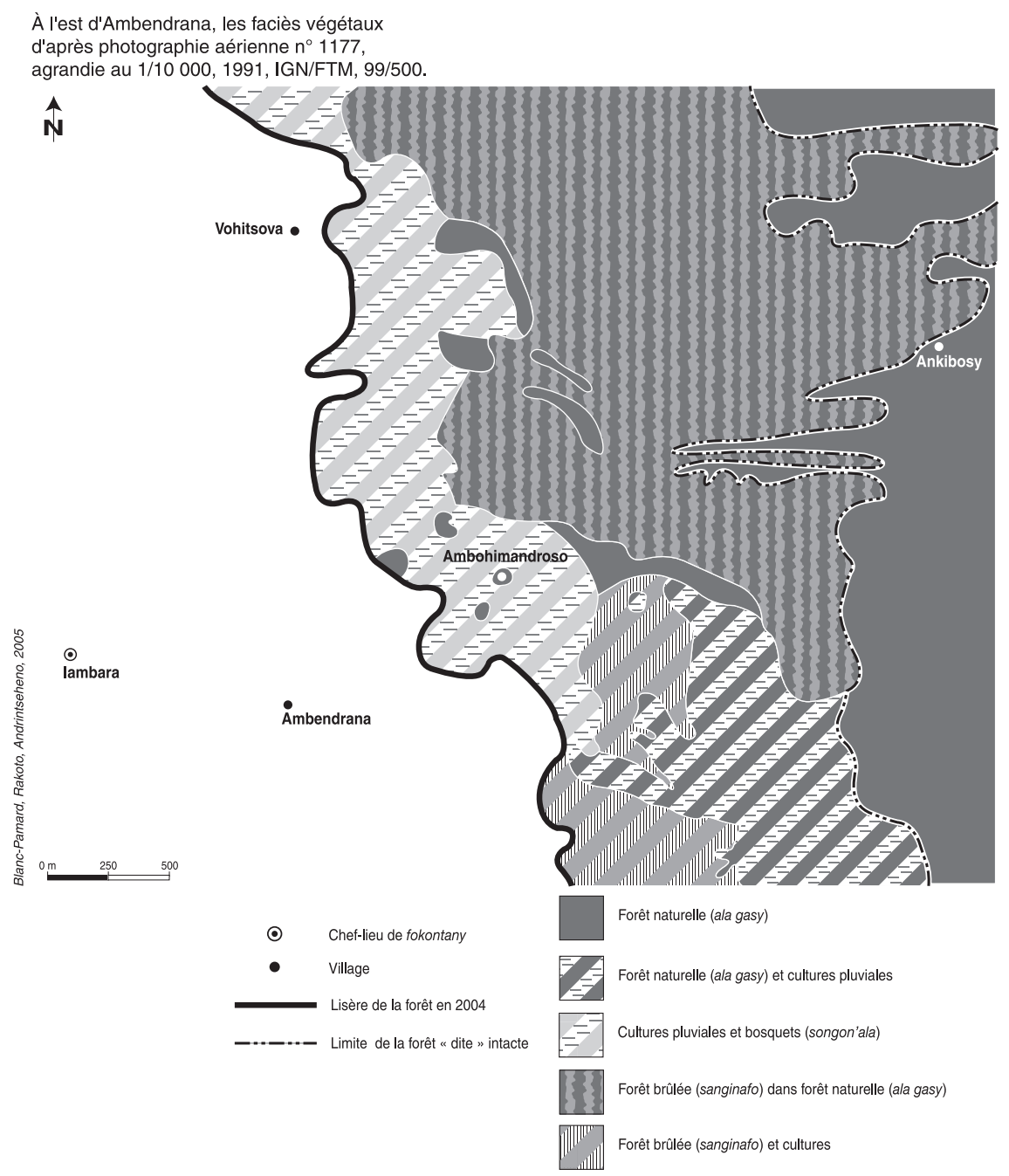

Fig. 4. Les faciès végétaux dans la forêt en GCF d'Ambendrana.

instances, formelles ou informelles, présentes au village : fokonolona ${ }^{15}$, église catholique, école, COBA...

À Amindrabe, prévaut une stratégie d'occupation des terres. Amindrabe est investi par des lignées territoriales, selon une logique de quadrillage territorial. Des « frères éclatés ", en concurrence selon leur lignée d'appartenance, se préoccupent d'humaniser un milieu déjà marqué de leurs droits. L'aménagement fait appel à une force de travail collective (haona, compagnie) que maîtrise la lignée sans pour autant rechercher une alliance avec les autres groupes, car le patrimoine foncier en cours de constitution n'est pas encore à partager.

Les deux logiques différentes sont liées à des profondeurs historiques inégales que confirment les généalogies : huit générations à Ambendrana, trois à Amindrabe, soit une logique d'enracinement pour l'un et une logique de territorialisation pour l'autre.

\footnotetext{
${ }^{15}$ Un groupe de descendance avec une communauté de
} résidence.
L'analyse des fonctionnements sociospatiaux dans les deux villages montre comment la gestion de la biodiversité est source de conflits : en gommant le foncier local et l'antériorité des droits des communautés, elle suscite une instrumentalisation des nouvelles règles foncières. Les conflits sont de deux ordres.

Les délimitations proposées au nom d'une gestion durable produisent un territoire inintelligible, voire illisible, que ce soit d'un point de vue écologique ou social. En effet, en réglementant les espaces à protéger et l'accès aux ressources, la contractualisation occulte le foncier local. Or, les limites patrimoniales (faritany, litt. limite de terre) ne sont pas visibles dans le paysage ou sont perceptibles des seules communautés locales; ce n'est pas le cas des limites récentes qui bornent le Parc national de Ranomafana ou les forêts contractualisées des GCF, identifiables aux marques de peinture rouge et aux numéros de même couleur sur le tronc des arbres. Les paysans grattent ces marques pour effacer les limites et la police des forêts doit contrôler ces marques. 


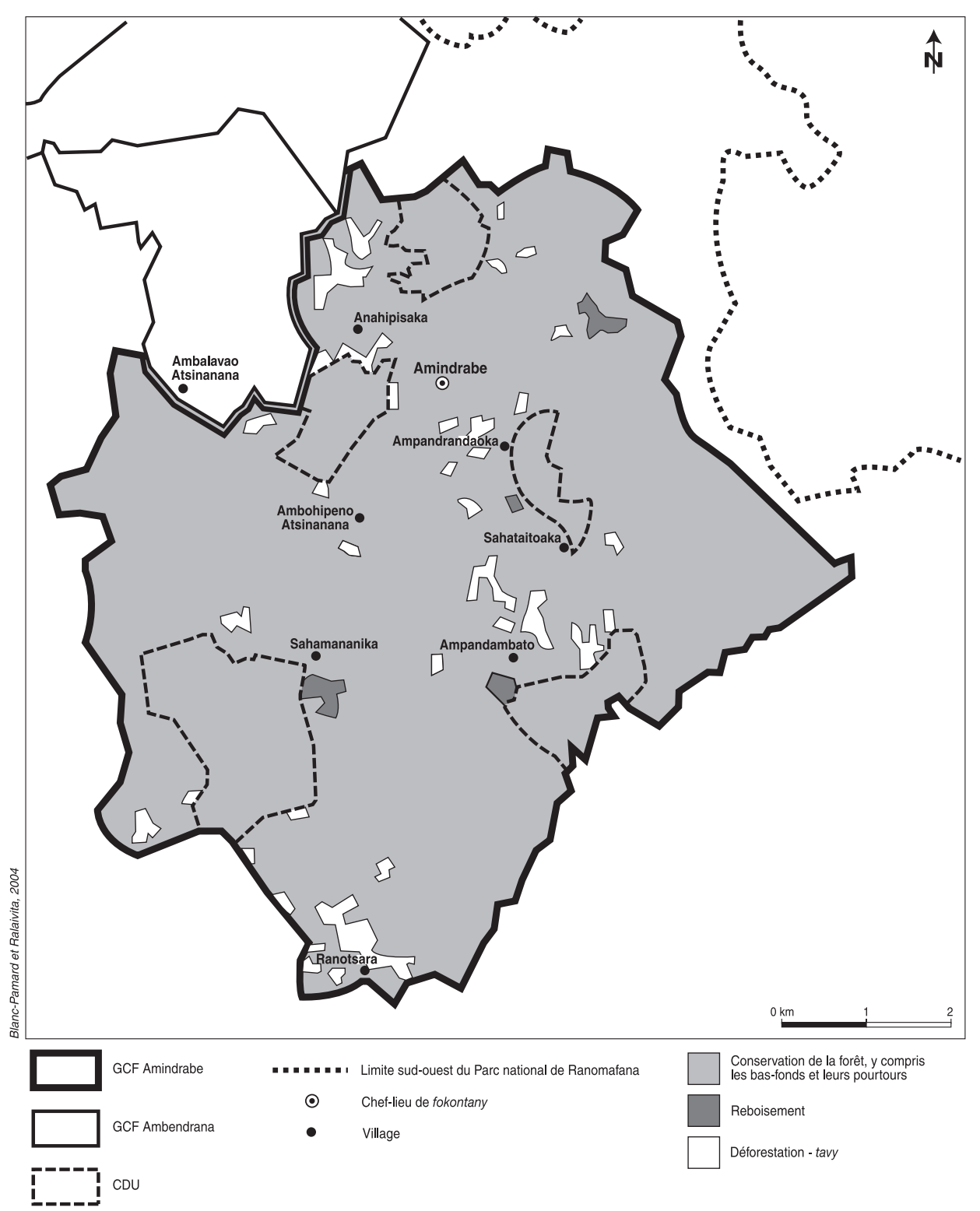

La superficie totale du périmètre forestier est de 5212,66 ha, soit les trois quarts du territoire du fokontany d'Amindrabe, répartie entre zone de conservation (3 396 ha) et zone de droits d'usage (1816,66 ha). La zone de droits d'usage (CDU, cantonnement des droits d'usage) représente $21 \%$ du périmètre forestier contre $43 \%$ dans et zone de droits d'usage
la GCF d'Andrendrana.

Fig. 5. Le zonage de la forêt gérée par la COBA d'Amindrabe.

Les « erreurs » de zonage sont une autre source de conflits. Les membres de la COBA d'Amindrabe ont été victimes d'une spoliation lors de la délimitation du périmètre de la GCF d'Ambendrana. Toute la partie nordouest de «leur » forêt leur a été dérobée, de même qu'une partie au sud-ouest (Fig. 8). Les membres de la COBA $\mathrm{d}^{\prime}$ Ambendrana reconnaissent d'ailleurs que le zonage du plan d'aménagement de la forêt n'est pas exempt d' « erreurs ", notamment l'annexion de la partie ouest du territoire $\mathrm{d}^{\prime} \mathrm{Amindrabe}{ }^{16}$. En fait, cette spoliation de terres

\footnotetext{
${ }^{16}$ Les délimitations ont été effectuées par des responsables des communautés locales qui ont accompagné les géomètres chargés du transfert de gestion.
}

apparaît être une stratégie affirmée d' « exclusion », pratiquée de longue date et avec assurance par Ambendrana.

Un autre problème concerne la localisation des différentes zones. Pour les forêts contractualisées d'Ambendrana (Fig. 7), la zone de droits d'usage actuels (2003-2005) est en partie comprise dans le territoire du groupe de descendance d'Ambohimandroso (taranaka), ce qui revient quasiment à en interdire l'accès au groupe de descendance de Vohitsova (taranaka), l'autre grand groupe historique du village. Par ailleurs, cette zone d'une superficie de 47 ha se trouve, en limite est, sur le territoire du groupe de descendance d'Amindrabe (lohona). Cette localisation excentrée n'encourage pas le respect des prélèvements en même temps qu'elle rend difficile 


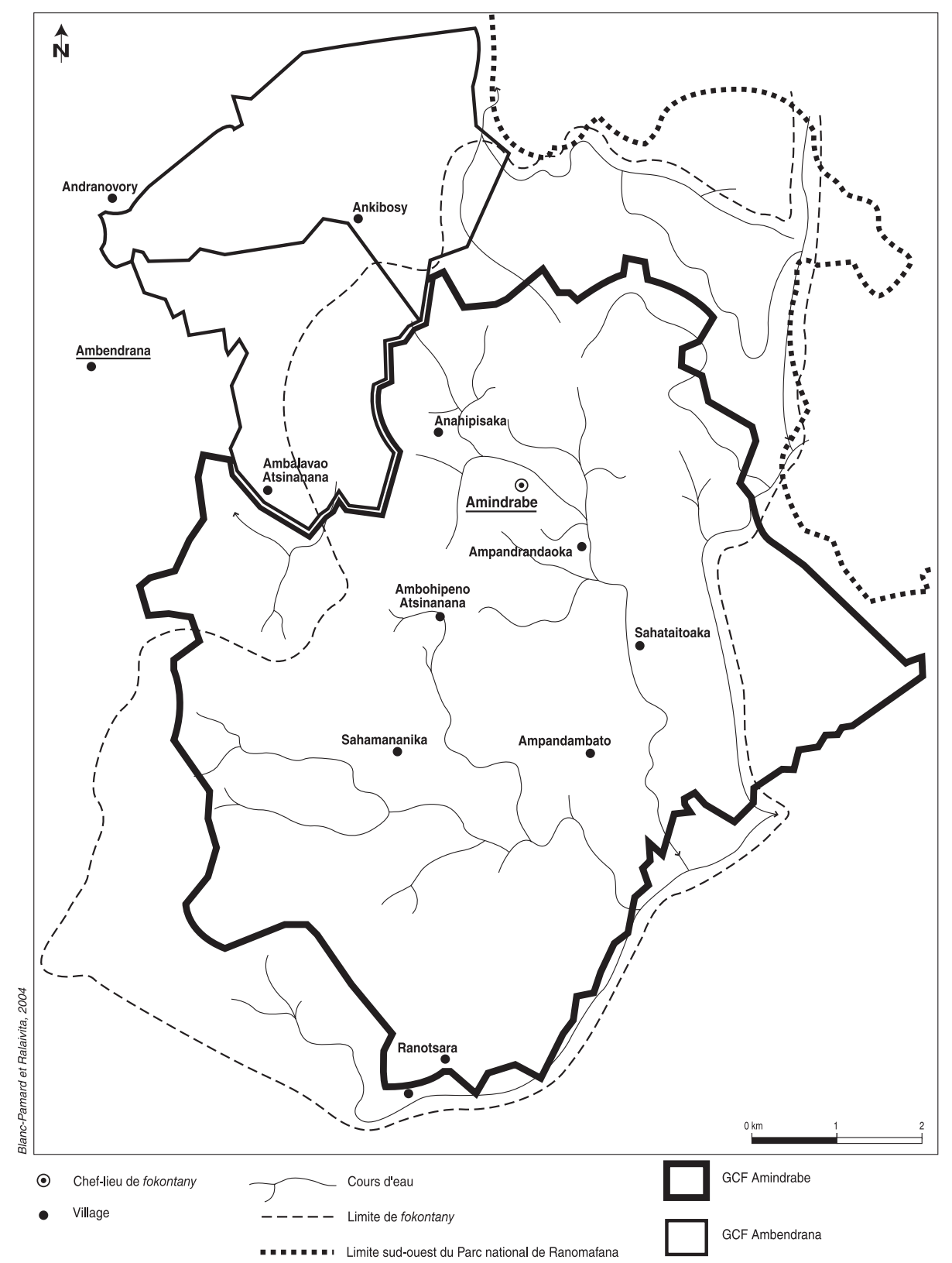

Fig. 6. Ambendrana et Amindrabe : une juxtaposition de territoires.

le contrôle des populations riveraines du fokontany voisin.

La capacité d'organisation des communautés rurales, postulat des environnementalistes à une gestion viable de la forêt, ne fonctionne pas dans le sens espéré, et reste au service d'objectifs qui ne visent pas les résultats attendus des politiques. Elle induit en effet le développement de stratégies de contournement par rapport au règlement et de stratégies d'appropriation foncière en accord avec le règlement. Cette instrumentalisation des nouvelles règles techniques et foncières entraîne une requalification des savoirs.

Les paysans sont conduits à modifier leurs pratiques dans le sens d'une protection de l'environnement. Loin d'être captifs de dispositifs réglementaires et loin de se laisser enfermer dans un système de contraintes (règles d'usage, d'accès, de production, de commercialisation), certains opèrent, soit individuellement, soit le plus souvent dans le cadre de leur groupe, une relecture des réglementations afin de les ajuster pour les transformer en opportunité technique, économique et/ou foncière. Cette catégorie de paysans peut être qualifiée d'écoopportuniste; c'est ce que pointent les deux exemples suivants de modification de pratiques et de mise au point de stratégies.

À Amindrabe : une stratégie foncière

La GCF donne l'autorisation de cultiver sur une largeur de $20 \mathrm{~m}$ au-dessus du bas-fond, le tavy (culture sur 


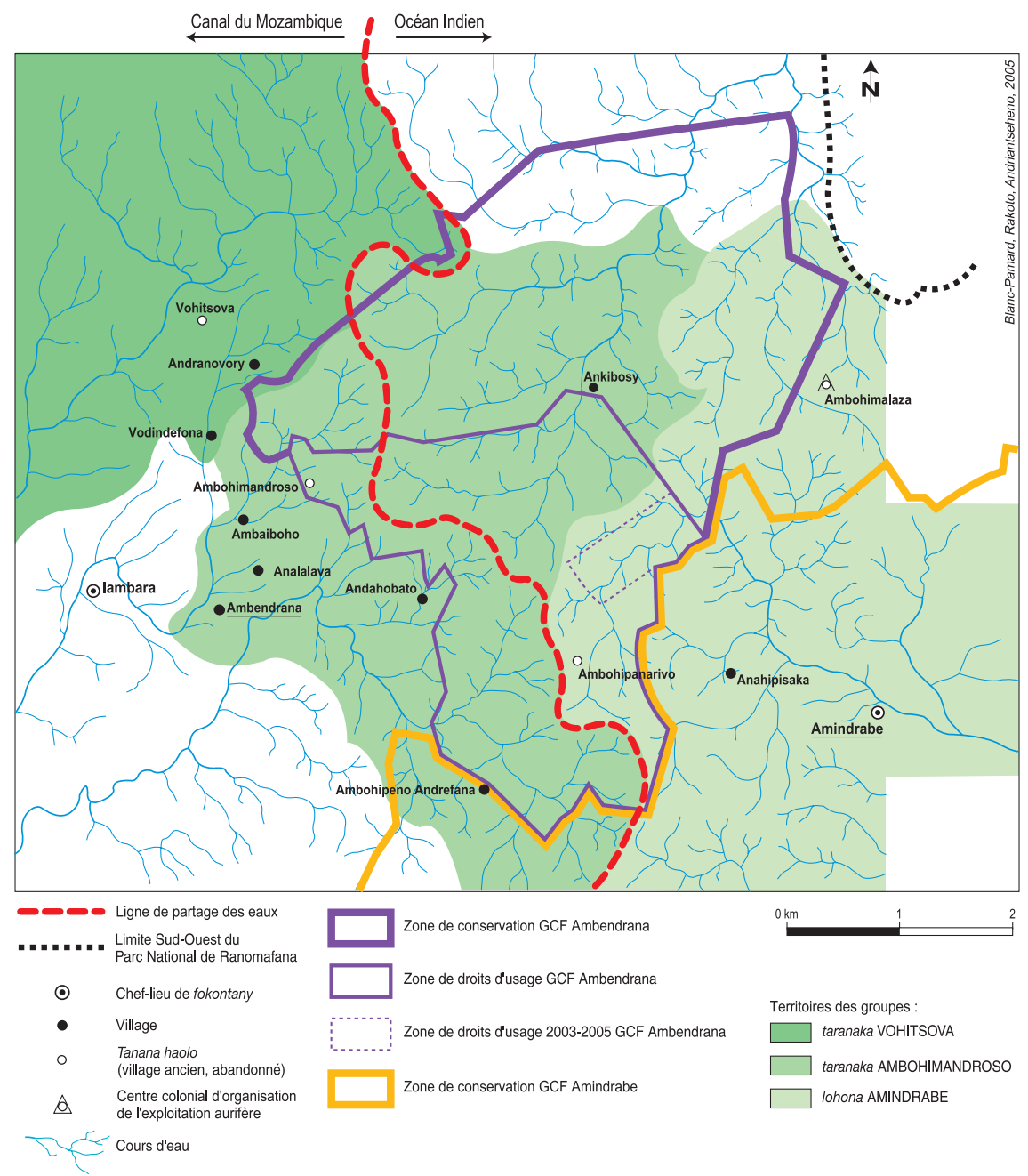

Fig. 7. Foncier local et foncier formel sur les territoires d'Ambendrana et d'Amindrabe.

abattis-brûlis) étant interdit au-delà de cette limite. Les mesures de conservation ont donc accéléré la mise en rizière et c'est à partir du bas-fond que la maîtrise foncière se conquiert (Fig. 9). Cette norme se traduit dans le paysage par une appropriation, avec la présence de plantes « résidentes » (pêcher, bananier, canne à sucre).

À Ambendrana : une stratégie économique et sociale qui se traduit par un contournement des normes techniques

L'autorisation de fabrication des manches d'angady ${ }^{17}$ a été, lors de la signature du contrat, limitée à six manches par exploitant pendant trois ans dans la zone de droits d'usage. Cette activité rémunératrice est, depuis longtemps, très développée pendant la période de soudure, notamment d'octobre à mi-décembre, à raison de 20 à 30 manches par artisan et par semaine, car la vente d'un manche permet d'acheter du riz.

Tous les hommes d'Ambendrana s'adonnent à cette production, dont le bénéfice est important et attendu.

\footnotetext{
17 Bêche à percussion lancée. Outil principal de l'agriculture malgache, celle-ci étant essentiellement manuelle.
}

Dans un tronc d'arbre, on découpe trois portions et, dans chacune d'elle, on fabrique 5 manches, soit 15 manches par tronc. La notoriété des forêts de cette région comme productrice de manches d'angady s'étend à toute l'île. L'exploitation à usage commercial est désormais interdite, car les normes d'exploitation forestière fixent le diamètre d'exploitabilité (dhp, diamètre à hauteur de poitrine) à $40 \mathrm{~cm}$ et les agents forestiers, lors de leur inventaire, n'ont pas trouvé d'arbres de ce diamètre ${ }^{18}$.

Le seul moyen de pouvoir continuer à exploiter les manches de bêche a été d'acculer le président de la COBA, un instituteur nouveau venu, à démissionner et d'élire à sa place le président de l'Association des artisans de manches d'angady. Les notables tiennent désormais le bureau de la COBA. Le changement dans les pratiques

\footnotetext{
18 Est-ce que la conservation de la biodiversité passe d'ailleurs par l'arrêt de l'exploitation? Les arbres dont le diamètre du tronc n'est que de $30 \mathrm{~cm}$ vont-ils grossir et à quelle échéance? Ceci a-t-il été pris en compte dans l'établissement de la norme dhp ? Sur ce thème de la production de normes d'écologisation, voir également Arnould et Glon (2005).
} 


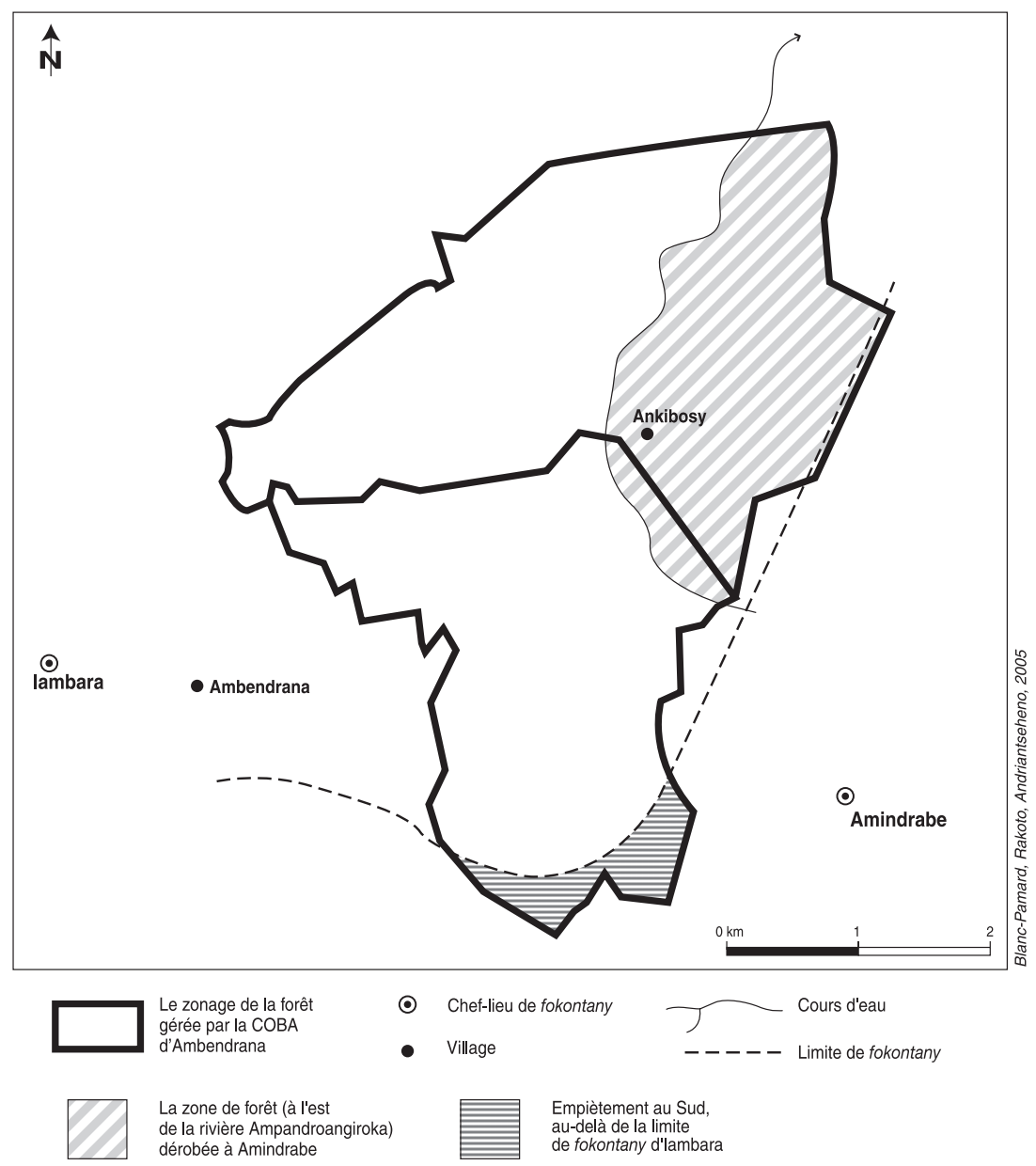

Fig. 8. Les « erreurs » de zonage dans la forêt gérée par la COBA d’Ambedrana.

Tableau. Comparatif de prix entre un manche d'angady et un kapoaka de riz.

\begin{tabular}{|l|lll|}
\hline & octobre $2003^{*}$ & octobre $2004^{*}$ & ${\text { octobre } 2005^{*}}^{*}$ \\
\hline 1 manche d'angady & 750 à 1000 FMG & 2000 FMG & 750 à 1200 FMG \\
\hline 1 kapoaka $^{* *}$ de riz & 750 FMG & 1150 à 1200 FMG & 1450 à $1500 \mathrm{FMG}$ \\
\hline soit environ & 287 à 330 g de riz & 487 g de riz & 230 g de riz \\
\hline
\end{tabular}

* Relevé des prix au marché d'Alakamisy Ambohimaha. En 2005, 12500 FMG = 1 euro.

** Kapoaka = boîte vide de lait concentré ; unité de mesure volumique correspondant à $287 \mathrm{~g}$ de riz blanc.

concerne la fabrication des manches et la commercialisation. L'exploitation continue en forêt où l'on voit des trouées signalant l'abattage des arbres. Mais les manches ne sont plus fabriqués sur place. Les troncs des arbres sont transportés au village, où des ateliers de production ont été installés dans les maisons où ne se faisait auparavant que la finition, c'est-à-dire le polissage. Pour la commercialisation, les vendeurs se sont organisés : les jours de marché, ils quittent ensemble le village vers 2-3 heures du matin et s'éclairent avec des torches en bambou. Il en est de même pour d'autres produits dont la commercialisation est interdite (paniers en bambou, écrevisses...).

À leur arrivée au marché, leur production est achetée en gros par des collecteurs de Fianarantsoa. La vente d'un manche d'angady permet d'acheter du riz. Mais si, entre octobre 2003 et octobre 2004, la différence d'augmentation entre le prix du riz et celui du manche a été favorable aux artisans, la situation s'est inversée l'année suivante. En effet, en octobre 2005, l'offre importante a entraîné une baisse du prix du manche d'angady et il fallait parfois deux manches pour acheter un kapoaka de riz (Tab.).

\section{«Gouverner l'environnement? »}

\section{De nouvelles règles du jeu}

Dans cette configuration évolutive, le transfert de gestion instaure de nouvelles règles du jeu. Quelques points sont à souligner. 


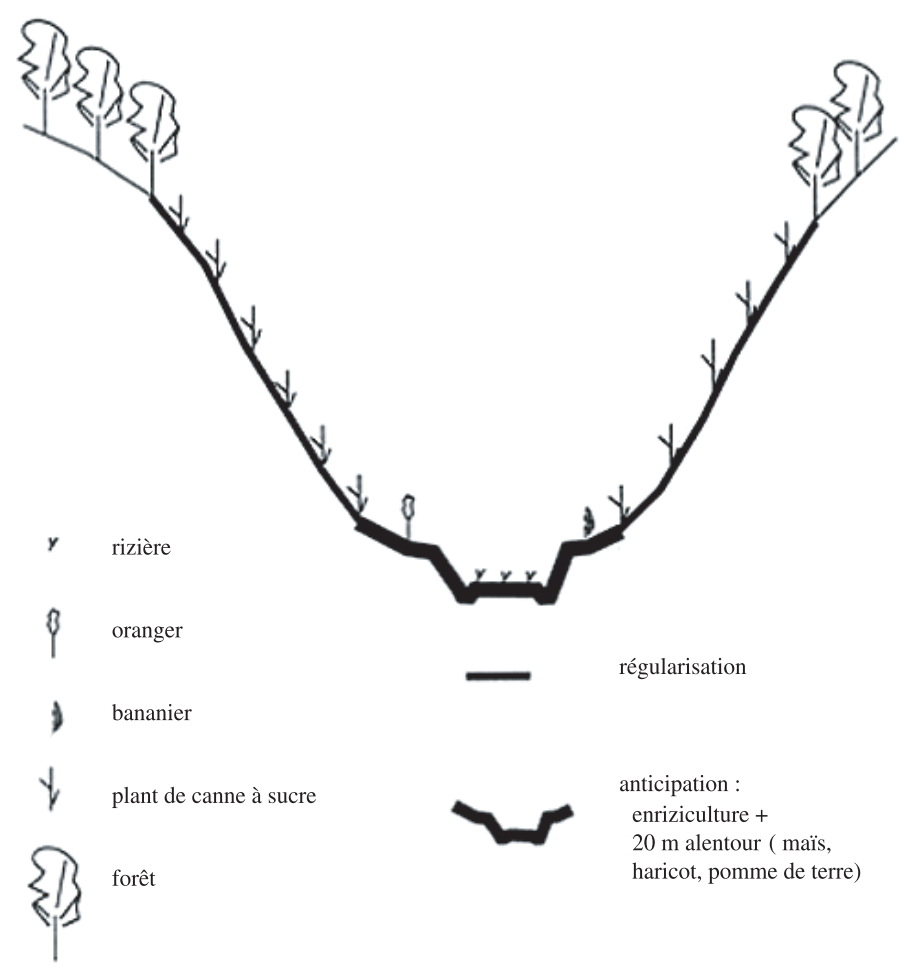

Fig. 9. GCF et pratiques foncières : légaliser, légitimer. Amindrabe : une traduction dans le paysage de la norme GCF.

Le cahier des charges précise que le défrichement de vingt mètres de part et d'autre du bas-fond est autorisé. Ceci entraîne de la part des paysans une double gestion du temps, soit par anticipation, soit par régularisation. La GCF n'est pas un transfert de propriété mais de compétence. En l'absence de sécurisation foncière, les paysans marquent la terre qu'ils défrichent aujourd'hui légalement - c'est-à-dire le bas-fond + $20 \mathrm{~m}$ - et celle qu'ils ont défrichée autrefois en plantant de la canne à sucre.

Le croquis montre ces deux stratégies foncières :

- dans le bas-fond et sur les versants adjacents, une anticipation avec enriziculture et défrichement sur la bande de $20 \mathrm{~m}$ autorisée (quitte à la déborder...) en installant des plantes résidentes (pêcher, oranger, bananier ou canne à sucre) et de petits champs avec des cultures de maïs, haricot, pomme de terre, patate douce... Un unique pied de pêcher ou d'oranger suffit à témoigner de l'appropriation du sol en bas de versant.

- sur les pentes autrefois défrichées par le tavy, une régularisation avec des plants de canne à sucre, marqueurs fonciers.

La forêt n'est pas qu'un écosystème, elle n'est pas simplement de dimension écologique; elle est une ressource locale commune et se définit comme un espace commun de pratiques et de savoirs. C'est aussi un élément d'un système social et territorial. Un schème comme la GCF est une projection à partir d'une représentation du monde qui repose sur un dualisme nature-société, alors qu'il s'agit d'un couple vital pour la dynamique $\mathrm{du}$ territoire. "Les terres d'ambala ${ }^{19}$ se marient avec les terres d'an'ala ${ }^{20}$ ", précisent les paysans. Pour les acteurs d'une politique publique environnementale, la forêt est une ressource rare et fragile, une réserve de biodiversité, dont le contrôle repose, d'une part, sur des normes d'exploitation forestière plus que sur une logique de gestion forestière durable, et, d'autre part, sur un zonage précisant le statut des espaces forestiers. En revanche, du point

\footnotetext{
19 «Là où se trouve le parc à bœufs ", c'est-à-dire le terroir aménagé, anthropisé.

20 «Là où se trouve la forêt », c'est-à-dire l'espace de la forêt «naturelle».
}

de vue des acteurs locaux, la forêt est une ressource complémentaire indispensable dans le cadre du système de production et de l'organisation sociale, une ressource qui ne se caractérise pas par sa rareté mais par sa place dans le système économique, social et territorial. Les paysans ne s'organisent pas par rapport à la rareté de la forêt, car, disent-ils, «quand il n'y en aura plus, on avisera ». La mise en ordre écologique du contrat GCF ne prend pas en compte les savoirs locaux, ne recoupe pas la mise en ordre sociale et ne considère pas le fait qu'ambala et an'ala sont complémentaires, à l'image d'un couple mari et femme. Le contrat de transfert de gestion sépare les deux unités et propose une autre alliance pour une durée déterminée dans la zone des droits d'usage actuels (47 ha pour Ambendrana), qui, on l'a vu, s'accorde mal avec l'organisation sociale.

Le foncier formel est en décalage par rapport au foncier local et crée une situation de pluralisme foncier. Les limites techniques de gestion durable s'ajoutent en discordance aux limites lignagères et administratives. La 
zone de conservation, noyau dur du plan d'aménagement où la protection est maximale, gèle le territoire forestier des paysans, puisque le seul moyen de prouver la mise en valeur d'une parcelle reste son défrichement par le droit de hache, ce qui explique que le bas-fond est désormais le point de départ de toute appropriation foncière. La GCF a ainsi accéléré une dynamique d'enriziculture dans le bas-fond et ses vingt mètres alentour, puisque les paysans installés en forêt se trouvent désormais en situation d'illégalité permanente. Non seulement la protection de la forêt entraîne des restrictions immédiates sur son usage, mais elle a aussi des conséquences à plus long terme en canalisant la stratégie foncière. Cette forme d'occupation limitée de l'espace autour du binôme bas-fond/versant sur vingt mètres renforce la logique agricole. L'objectif paysan n'obéit pas, en effet, à une logique forestière - $c^{\prime}$ est-à-dire une reconstitution de la forêt (la durée de la jachère étant la clé du système) -, mais, au contraire, vise, par une succession des cultures, à obtenir un sol défriché et débarrassé des ligneux en mesure de devenir une rizière.

La GCF est un transfert de compétences et non de propriété. C'est pourquoi l'absence de sécurisation foncière active une consolidation des patrimoines fonciers. À Ambendrana, une logique d'extension par exclusion, achat de terres ou alliance matrimoniale est à l'œuvre. À Amindrabe, prévaut une logique territoriale sans recherche d'alliance : il s'agit de marquer et de gagner des terres au niveau des segments de lignée. La rizière rend possible la stabilité de l'habitat et, ainsi, un élargissement de l'espace social.

L'objectif de la GCF consiste à valoriser le territoire pour en préserver les ressources naturelles. On constate pourtant que, depuis l'intervention du programme, une privation des richesses locales et une paupérisation croissante marquent la majorité des populations du corridor. «La forêt qui était leur richesse » leur a échappé en devenant corridor et enjeu national. Une logique de gestion imposée au niveau international est en contradiction flagrante avec la perception locale d'une ressource, héritage ancien et essentiel à la survie quotidienne : le lova lovana, le patrimoine hérité.

Une telle orientation des politiques environnementales ne peut se faire sans le relais des lettrés, médiateurs auprès $\mathrm{d}^{\prime}$ une population à fort taux d'illettrisme. Les instituteurs se retrouvent présidents des COBA sans pour autant bénéficier de l'autorité nécessaire, d'où une fragilité sur laquelle jouent les "notables». Dans les deux COBA, les présidents de l'association, un lettré et un nouveau venu ${ }^{21}$ (vahiny) dans les deux $\operatorname{cas}^{22}$, sont dépourvus de légitimité auprès des populations locales et se trouvent confrontés à des oppositions ouvertes ou

\footnotetext{
21 Jusqu'en décembre 2004 pour la COBA Ambendrana.

22 Il s'agit d'instituteurs, de l'école privée catholique à Ambendrana, de l'école publique à Amindrabe.
}

sournoises. On peut même se dire que la stratégie des autochtones d'élire un étranger, par définition «non durable ", est contradictoire avec l'engagement au principe de «gestion durable des ressources forestières ${ }^{23}$ ».

\section{Des territoires de conservation aux territoires de gestion}

Les dynamiques observées à Ambendrana et à Amindrabe soulignent ici que les politiques agissant sur les territoires et les communautés pèchent dans leur mise en œuvre sur deux points : l'illusion participative (BlancPamard et Fauroux, 2004); la maille de gestion environnementale.

\section{L'illusion participative}

La politique de transfert de gestion des ressources repose sur le potentiel d'organisation des communautés. Cependant, ce dernier ne fonctionne pas comme attendu et reste mobilisé en vue d'objectifs qui ne sont pas ceux de la conservation. La gestion a créé du participatif par une structure socio-organisationnelle, celle de l'association COBA, qui reconstitue ou prolonge en fait des micropouvoirs locaux autour des notables.

La COBA est une association de paysans créée en vue d'assurer la gestion participative des forêts avec le service des Eaux et Forêts. Le transfert de gestion a été perçu par les paysans comme un retour, ou même une renaissance, des liens avec la (leur) forêt. C'est une « récupération » ou encore une réappropriation de la forêt "comme avant», sous-entendu gérée par les villageois et en accès " dit » libre, c'est-à-dire selon leurs normes.

\section{La maille de gestion environnementale}

La «bonne échelle » (ou la maille d'action) du transfert de gestion reste à définir. L'enjeu est d'identifier des configurations sociospatiales locales qui peuvent être porteuses d'une gestion de l'environnement et qui s'articulent avec les autres territoires présents. La question de la cohabitation d'espaces protégés et d'espaces productifs est une question importante, comme celle du gabarit adéquat pour le transfert de gestion. La recherche du bon territoire de gestion pose également celle du bon usage des ressources naturelles renouvelables.

Une "gestion de la confusion ${ }^{24}$ " prévaut là où un transfert de gestion est censé clarifier les responsabilités des populations riveraines et les apports dont celles-ci devraient bénéficier en relation avec l'administration forestière, leur partenaire. Faut-il voir sur ce point la traduction d'une limite de l'approche GCF, qui ne s'occupe pas

\footnotetext{
23 Obligations des COBA, alinéa 5.

24 Expression empruntée à Paul Mathieu (1996).
} 
de la dimension foncière du transfert, alors que l'accès à la ressource est conditionné par l'accès au foncier, dans le même temps où la réforme malgache en cours ${ }^{25}$ entend instaurer une gestion foncière favorable « à la gestion, la protection, la restauration et [au] renouvellement des ressources naturelles ${ }^{26} »$ ? Il faut aussi poser la question des cadres adéquats pour le transfert de gestion : terroir, fokontany, commune..., qui entrent parfois en concurrence en l'absence de concertation. Une gestion localisée de l'espace implique une mise en contexte pour comprendre les incompatibilités des référentiels entre les différents niveaux d'acteurs concernés. La logique de bassin versant invite ici à trouver l'élément transcendant les échelles pour en démêler la complexe imbrication.

À terme, l'évolution de la nature ambiguë des transferts de gestion demeure susceptible de se faire dans des dimensions divergentes suivant la manière dont les différents acteurs locaux se l'approprient.

\section{Conclusion}

Les TGRNR conduisent à la fois à la délimitation de nouveaux territoires et à la modification des rapports complexes entre les différents acteurs. Ce constat rend nécessaire l'analyse des relations entre savoirs, pouvoirs et territoires des différents acteurs avant toute tentative de " gouverner l'environnement » (Blanc-Pamard et Rakoto Ramiarantsoa, 2003). Bien qu'une plus grande souplesse marque le processus GCF depuis $2001^{27}$, par rapport au processus GELOSE, le transfert de gestion ne semble pas constituer une étape vers un changement institutionnel visant à un objectif de conservation de la biodiversité. Même si des recompositions sont observées, il est bien plutôt un moyen de renforcer le statu quo.

L'analyse nous conduit à proposer que, pour souscrire à un tel objectif, les démarches de gestion territoriale impliquent l'ensemble des acteurs concernés par les processus de redéfinition de l'usage de la forêt (réglementations, obligations, droits, restrictions...). Par ailleurs, l'étude menée dans deux villages révèle combien le corridor RAI se présente actuellement comme un archipel de territoires locaux de conservation dont la juxtaposition et la superposition, mises en évidence par les disjonctions spatiales des zones délimitées, posent la question des cohérences territoriales et se prêtent à de nombreuses

\footnotetext{
${ }^{25}$ Loi n $^{\circ} 2005-019$ du 17 octobre 2005, qui fixe les principes régissant les statuts des terres.

26 Programme national foncier. Document de présentation du ministère de l'Agriculture, de l'Élevage et de la Pêche (MAEP) ; voir les différentes mises à jour entre octobre 2004 et décembre 2005 sur le site Internet du ministère :

http://www.maep.gov.mg/fr/actupnfaccueil.htm

${ }_{27}$ Contrat bipartite (et non plus tripartite), absence de médiateur environnemental, sécurisation foncière relative abandonnée.
}

formes de contournement. Ces îlots ne configurent pas les éléments d'un réseau de conservation de la biodiversité de la forêt impliquant des échelles et des niveaux multiples d'organisation. Un projet régional visant à rendre fonctionnel le corridor nécessiterait un pacte territorial fédérant les différents types d'acteurs concernés, une politique de conservation plus participative et reconsidérant la juxtaposition des territoires protégés et des espaces productifs.

Les politiques de conservation de la biodiversité mobilisées ici génèrent des situations conflictuelles et des stratégies de contournement, car leur mise en œuvre différenciée procède de logiques spécifiques et produit de nouvelles organisations territoriales et sociales. Sans prise en compte des réalités locales, elles n'évitent pas un décalage entre conservation biologique et territoires sociaux. Les limites des entités concernées reposent en effet sur des critères de nature différente : critère de 1 '«objectivité » scientifique naturaliste pour la conservation; critère de l'appropriation lignagère d'un espace de ressources pour l'entité sociale. En modifiant, réglementant ou interdisant des activités considérées comme néfastes, via l'établissement de mesures réglementaires ou la délimitation de zones particulières, c'est bien l'articulation entre développement et environnement qui est en cause et qui pose la question des coordinations territoriales à promouvoir et des dispositifs entre acteurs et opérateurs. "Gouverner l'environnement » réunit ainsi les attributs d'une politique publique dont l'apprentissage est en cours et qui soulève des questions à partir des évaluations scientifiques des ressources à conserver, des échelles de temps et d'espace, de la prise en compte des modes locaux d'accès et d'usage des milieux, des stratégies et des besoins des acteurs.

\section{Références}

Andriananja, H., Raharinirina, V., 2004. Quels enjeux pour la durabilité et la gouvernance des ressources naturelles et forestières à Madagascar?, Mondes en développement, 32, 3, 91-99.

Arnould, P., Glon, E. (Eds), 2005. La Nature a-t-elle encore une place dans les milieux géographiques?, Paris, Publications de la Sorbonne.

Aubert, S., 2002. La gestion patrimoniale des ressources forestières à Madagascar : limites et perspectives d'une "révolution par le haut ", in Cormier-Salem, M.-C., et al. (Eds), Patrimonialiser la nature tropicale, Paris, IRD Éditions.

Berlan, J.-P., 2002. Les « enclosures » du vivant, in Vivien, F.-D. (Ed.), Biodiversité et appropriation : les droits de propriété en question, Paris, Elsevier/NSS, 39-70.

Blanc-Pamard, C., Rakoto Ramiarantsoa, H., 2003. Madagascar : les enjeux environnementaux, in Lesourd, M. (Ed.), L'Afrique : vulnérabilités et défis, Nantes, Éditions du Temps, 354-376.

Blanc-Pamard, C., Fauroux, E., 2004. L'illusion participative. Exemples ouest-malgaches, Autrepart, 31, 3-19. 
Blanc-Pamard, C., Ralaivita, M., 2004. Ambendrana : un territoire d'entre-deux. Conversion et conservation de la forêt (corridor betsileo, Madagascar). Rapport GEREM, IRD-CNRE / CNRSEHESS CEAf, Paris.

Blanc-Pamard, C., Rakoto Ramiarantsoa, H., Andriantseheno, D., 2005. Foncier et territoires entre pouvoirs locaux et politiques publiques environnementales : pratiques, acteurs, enjeux (corridor betsileo, Madagascar). Raport GEREM, IRD-CNRE / CNRS-EHESS CEAf / ICOTEM, Université de Poitiers, Paris.

Burel, F., Baudry, J. 1999. Écologie du paysage : concepts, méthodes et applications, Paris, Lavoisier.

Carrière-Buchsenchutz, S., 2006. L'urgence d'une confirmation par la science du rôle écologique du corridor forestier de Fianarantsoa, Études rurales, 178, 181-196.

Cleaver, K.M., Schreiber, G.A., 1994. Reversing the Spiral: The Population, Agriculture, and Environment Nexus in SubSaharan Africa, Washington (DC), World Bank.

Da Lage, A., Métailié, G., 2000. Dictionnaire de biogéographie végétale, Paris, CNRS Éditions.

Forman, R.T.T., Godron, M., 1981. Patches and structural components for a landscape ecology, BioScience, 31, 733740.

Fremigacci, J., 1998. La forêt de Madagascar en situation coloniale : une économie de la délinquance (1900-1940), in Chastanet, M. (Ed.), Plantes et paysages d'Afrique: une histoire à explorer, Paris, Karthala / Centre de recherches africaines, 410-439.

Freudenberger, M., Freudenberger, K., 2002. Contradictions in agricultural intensification and improved natural resource management: Issues in the Fianarantsoa forestcorridor of Madagascar, in Barrett, C.B., Place, F., Aboud, A.A., Natural Resources Management in African Agriculture: Understanding and Improving Current Practices, Wallingford,
CABI Publishing / International Centre for Agroforestry, 181-192.

Giraut, F., Guyot, S., Houssay-Holzschuch, M., 2004. Les aires protégées dans les recompositions territoriales africaines, L'Information géographique, 68, 340-368.

Goodman, S.M., Benstead, J.P. (Eds). 2003. The Natural History of Madagascar, Chicago, University of Chicago Press.

Jarosz, L., 1996. Defining Deforestation in Madagascar, in Peet, R., Watts, M. (Eds), Liberation Ecologies: Environment, Development, Social Movements, New York, Routledge, 148164.

Marcus, R.R., Kull, C.A. (Eds), 1999. The Politics of Conservation in Madagascar, African Studies Quarterly, 3, 2.

Mathieu, P., 1996 Pratiques informelles, gestion de la confusion et invention du foncier en Afrique, in Villers, G. de (Ed.), Phénomènes informels et dynamiques culturelles en Afrique, Bruxelles/Paris, Institut africain-CEDAF / L'Harmattan, 6478.

PE III, 2005. Évaluation et perspectives des transferts de gestion des ressources naturelles renouvelables (TGRNR). Rapport, Ministère de l'Environnement, des Eaux et Forêts, Antananarivo.

Rakoto Ramiarantsoa, H., Samyn, J.-M., 2004. Arrimer le local et le global, ou le développement durable pour qui? L'exemple de la gestion contractualisée de la forêt de Merikanjaka, Mondes en développement, 32, 127, 91-99.

Rakotosamimanana, B., Ganzhorn, J., 1995. Rapport final de l'Atelier scientifique sur la définition des priorités de conservation de la diversité biologique à Madagascar, 10-14 avril 1995. Projet PRIF/GEF, ONE, DEF, ANGAP, PNUD, Conservation International, Antananarivo.

Ramamonjisoa, B., 2004. Origines et impacts des politiques de gestion des ressources naturelles à Madagascar, Schweiz. Z. Fortswes., 155, 11, 467-475.

Reçu le 13 janvier 2006. Accepté le 3 mai 2007. 